\title{
Modeling astronomy education, the case of F-HOU tools : SalsaJ and Human Orrery
}

\author{
Emmanuel Rollinde* \\ CY Cergy Paris Université, LDAR, \\ F-95000 Cergy, France \\ Universités de Paris, Artois, \\ Paris-Est Créteil, Rouen
}

accepted for publication in the proceeding of

Education and Heritage in the era of Big Data in Astronomy Proceedings IAU Symposium No. 367,

2020. R.M. Ros, B. Garcia, S. Gullberg,

J. Moldon 83 P. Rojo, eds., Dec 2020 


\begin{abstract}
This communication introduces two cases of the use of astronomy as a motivating context to interdisciplinary science education with emphasis on modeling activities. Firstly, a dedicated software, called SalsaJ, allows students to reproduce the same data analysis as made by astronomers. The case of exoplanet detection will be used as an exemple. Secondly, bodies of learners are considered to model movements of planets with a Human Orrery (a Spatio-Temporal Map of the Solar System), connecting thus mathematics, physics, geography and arts.
\end{abstract}

Keywords: methods: data analysis, solar system: general, astronomy education

\title{
INTRODUCTION
}

The association F-HOU is part of a worldwide project, Global Hands-On Universe[13], that aims to promote a method of learning sciences through astronomy. We are a community of science teachers, astronomy researchers and science education researchers, whose main goal is to provide science and mathematics teachers from middle school to high school with interactive and collaborative tools (software, instruments, methodology, documents shared on the Internet). Based on an investigative pedagogy that emphasizes observation, the global objective is to develop students' curiosity, to maintain their taste for science and technology, and to lead them to argue and reason [8]. This approach requires interaction between physical sciences and mathematics, and can also be easily integrated into multidisciplinary teaching (combining literature, philosophy, technology, language teaching and sports).

\section{MODELING ACTIVITIES AND ASTRONOMY}

Our pedagogical approach is based on the principle of modeling and the resulting link between mathematics and physical sciences $[3,4]$. To address and reflect on a problem, an expert or student will use two registers as described in [11] and [10], see Fig. 1 (right): the empirical register which consists of objects, phenomena and actions on objects and phenomena; the theoretical register which corresponds to an abstraction applicable to any real object. As for students, the theoretical register corresponds to a set of statements that the student holds to be true and that allow him or her to make sense of the real. A model can then be considered as a link between these two registers. Modeling activities make students 
work from one register to another, or within one register. Modeling in the context of mathematics education makes a specific emphasis on horizontal and vertical mathematization $[1,12]$. The first is the transformation of the real model into a mathematical model, which requires to choose a set of physical parameters. The second is the obtaining of a mathematical result that can be later on translated into real results, or a description of the real situation.
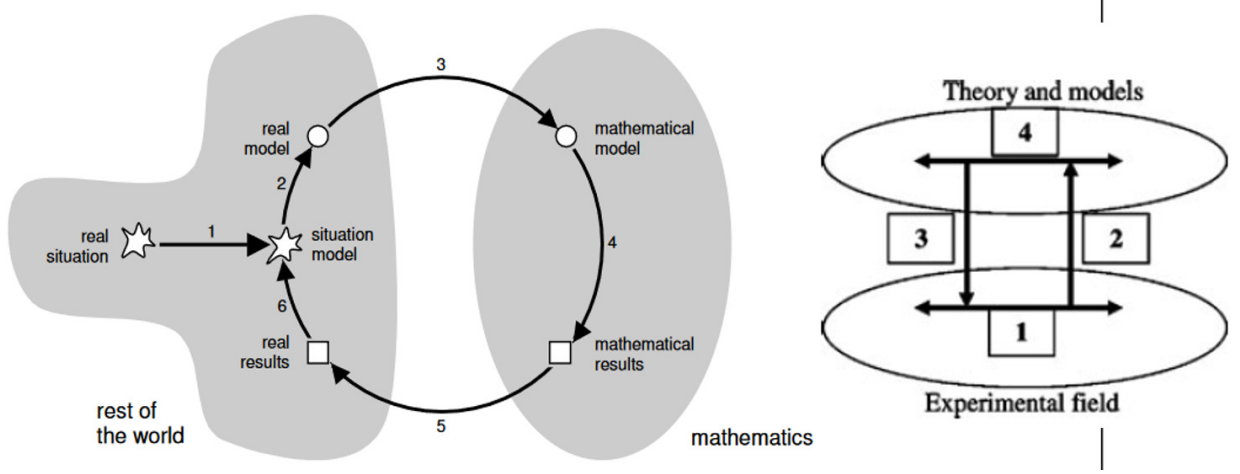

FIG. 1. Two illustrations of modeling process. left: the modeling cycle presented in [1]. right: the theory of the two worlds presented in [10]. See main text for application to astronomy in education.

Astronomy provides teachers and students with real situations that are very specific. First, the real situation in astronomy is a 'single case' situation, meaning that one cannot modify the observed celestial objects, or move around to see them from different point of view. In most cases (at least in the educational context), few objects will be observed to infer general laws. This was the case for Kepler and Newton who had to infer classical mechanics relations and laws from the observation of one single Solar System. This is less the case today with the era of big data even in astronomy. Second, real situation is observed through the light send or reflected by the objects. Hence, the situation given to the learner is already a model of the real situation provided by either an image or a spectrum. Making sense of this modeled situation is not straightforward and imposes the use of modeling cycle. Based on astronomy, and compared to 'everyday-situations' [12] students' activities will enhance two aspects of modelisation: inferring the right model that should relate best to the real situation; choosing physical parameters that can be measured in the modeled situation (the image) and that can later on make sense of the real situation (after the two mathematizations). 


\section{MODELING ACTIVITIES WITH DATA ANALYSIS}

To make data manipulation and measurement feasible in the class, a specific software, SalsaJ, has been developed in Java $[2,6]$. Its acronym stands for "Such A Lovely Software for Astronomy in Java". Downloaded free of charge from the project site, it works on all platforms and can be used in different European languages as well as in Arabic and Chinese. The pedagogical sequence we present here is based on the detection of an exoplanet through its transit in front of its host star. The students have at their disposal a time series of images (Fig. 2, left) which accounts for their empirical register. The objective is to find clues revealing the presence of a planet in orbit around a star.
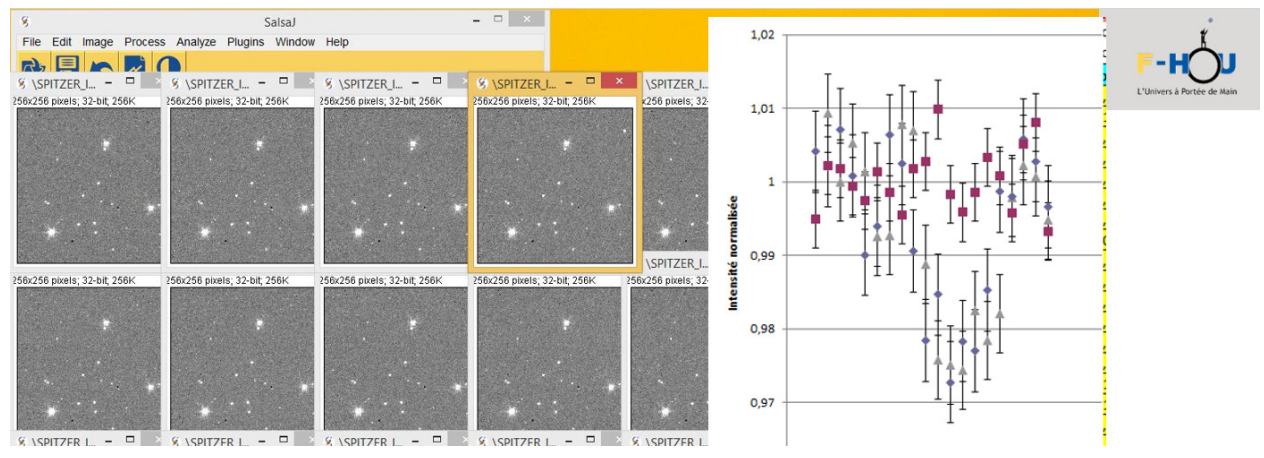

FIG. 2. Modeling activities on the detection of an exoplanet. The real problem is provided by a series of image of the same field taken at regular time interval (left). The model used is that of a star and an exoplanet orbiting around it. The mathematical prediction of the model is a graph showing the evolution with time of the luminosity of different stars in the field (right).

A first modeled situation of a star and an exoplanet must be derived through the understanding that the light received by the observer (empirical register) is emitted by point stars (theoretical register). The learner must then use this model for predicting that the planet blocks part of the light when it is located between the observer and the star (theoretical register). Through this first cognitive task that connects two registers, the photometry emerges as a good candidate to use the model for making sense of the observed images and to make measures. Once the students proceed and make the measure using the tools provided by the software, they end up with a mathematical representation of the evolution of the luminosity of three different stars with time (Fig. 2, right) that may be compared to the prediction. The mathematical resolution consists in a statistical analysis of the significance of the departure 
from a constant luminosity for one of the series of data (or one of the star). The modeling cycle ends by connecting the amplitude of the observed decrease to the ratio of the surface of the star versus that of the planet.

\section{MODELING WITH THE LEARNING BODY}

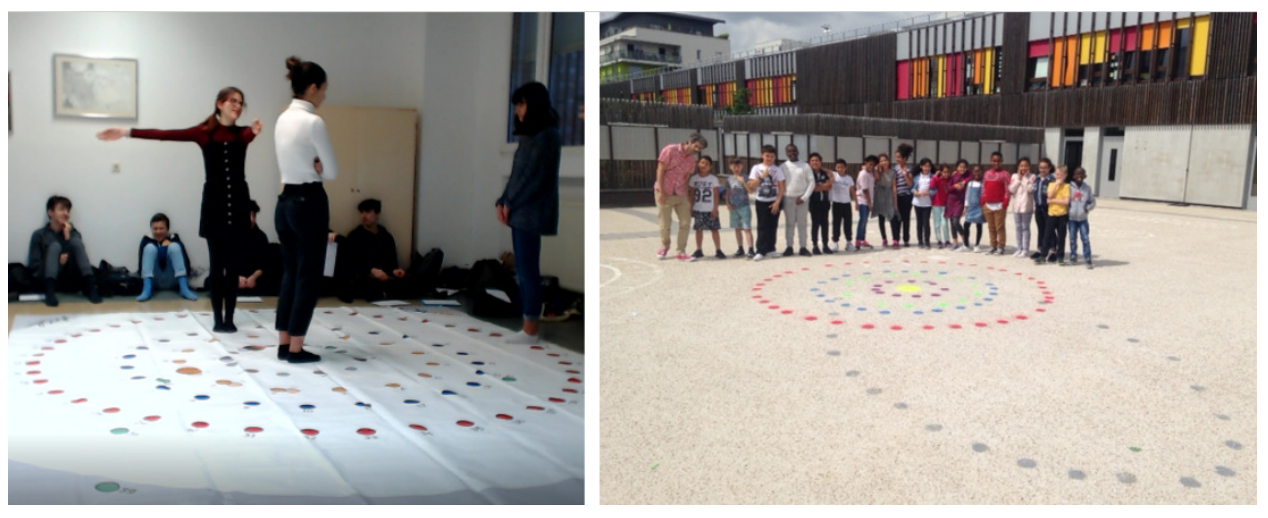

FIG. 3. Modeling activities on the Solar System. Students enact the movements of planets and comets around the Sun on a 2D representation of the orbits, a so called Human Orrery. Left: Students enact and observe movements as seen from different referential frames. Right: Primary school children in front of the Human Orrery they have build in their courtyard.

A "human Orrery" (Fig. 3) allows the learners to enact the planets' movement with correct relative speed. An Orrery is a mechanical or digital device designed to model the motion of the planets around the Sun and their changing positions in the sky. On a human Orrery, the orbits of planets and comets are drawn at a human scale allowing movements in the Solar System to be enacted by the learners $[5,7]$. We give here one example of how this tool may be used to help students learn practice modelisation [9].

All grade 10 students of a French high school were involved in two successive teachinglearning sessions. The learning material consisted of two versions of the Human Orrery. One was an A4 model used individually on a table, with reference frames and trajectories drawn on a translucid paper; the other was a Human size version, with reference frames and celestial bodies enacted by students' bodies. Students enacted movements with their fingers and with their bodies respectively. Activities illustrated the movements of Earth, Mars, and Sun during 24 hours and one year, observed from different reference frames (terrestrial, 
geocentric or heliocentric). Students had to use the model for predicting trajectories in each referential frame. To do so, they enacted the trajectories, observed them, and discussed whether length and speed of the planets were different when observed in each referential frame. By doing so, they also proceeded through the modeling cycle, had to make predictions and test them. Hence, the model of the Human Orrery helps to make sense of what happens to a trajectory during a change of referential frame.

\section{CONCLUSION}

This communication intends to motivate and enhance the explicit reference and use of modeling activities in the field of astronomy education. The set up and evaluation of such activities requires to combine expertises from professional astronomers, didactics researchers (in mathematics and science) and teachers. In January 2021, a francophone conference[14] has gathered 200 people coming from those three communities. We are confident that the international effort led by UAI on astronomy education will be fruitful in the set up of this community.

* emmanuel.rollinde@cyu.fr

[1] Blum, W., \& Leiss, D. 2007, Mathematical Modelling. Education, Engineering and EconomicsICTMA, 12, 222-231.

[2] Doran, R., Melchior, A. L., Boudier, T., Ferlet, R., Almeida, M. L., Barbosa, D., \& Roberts, S. 2012, arXiv preprint arXiv:1202.2764.

[3] Helding, B., Megowan-Romanowicz, C., Ganesh, T., \& Fang, S. 2013, in Modeling Students' Mathematical Modeling Competencies, 327-339, Springer, Dordrecht.

[4] Hestenes, D. 2006, Proceedings of the 2006 GIREP conference: Modeling in physics and physics education, 31, 27. Amsterdam: University of Amsterdam.

[5] Rollinde, E. 2016, International Journal of Science and Mathematics Education, 17(2), 237252.

[6] Rollinde, E., Ferlet, R., Melchior, A. L., Delva, P., Chagnon, G., \& Salomé, P. 2016, Le Bulletin de l'Union des Professeurs de Physique et de Chimie, 4(983), 469-496. 
[7] Rollinde, E., \& Decamp, N. 2019, Journal of Physics: Conference Series, IOP Publishing, 1287(1), 012011.

[8] Rollinde, E., Pennypacker, C., Doran, R., Darhmanoui, H., Handa, T., Kothari, K., Lewis, F., Robberstad, J., \& Megowan, C. 2020, Futures of Education - GHOU 2020, Intern report, hal-03089436

[9] Rollinde, E., Decamp, N., \& Derniaux, C. 2021, Physical Review Physics Education Research, submitted

[10] Sensevy, G., Tiberghien, A., Santini, J., Laubé, S., \& Griggs, P. 2008, Science education, 92(3), 424-446.

[11] Tiberghien, A. 1994, Learning and instruction, 4(1), 71-87.

[12] Yvain-Prébiski, S., \& Modeste, S. 2020, Proceedings of the CIEAEM conference, 71, 139-150.

[13] Http://handsonuniverse.org/france.

[14] AstroEdu-FR, https://astroedu-fr.sciencesconf.org/ 